\title{
A New South Polar Digital Terrain Model of Mars from the High Resolution Stereo Camera (HRSC) onboard the ESA Mars Express
}

\author{
Alfiah Rizky Diana Putri ${ }^{\mathrm{a}, *}$, Panagiotis Sidiropoulos ${ }^{\mathrm{a}}$, Jan-Peter Muller ${ }^{\mathrm{a}}$, \\ Sebastian H. G. Walter ${ }^{\mathrm{b}}$, Greg G. Michael $^{\mathrm{b}}$ \\ ${ }^{a}$ Imaging Group, Mullard Space Science Laboratory, University College London, Holmbury \\ St. Mary, Dorking, Surrey, RH5 6NT, UK \\ ${ }^{b}$ Freie Universität Berlin, Department of Geosciences, Institute for Geological Sciences, \\ Planetary Sciences and Remote Sensing, Malteserstr. 74-100, 12249 Berlin, Germany
}

\begin{abstract}
The first high-resolution Digital Terrain Model (DTM) of the entire South Pole of Mars has been produced. A modified version (Kim and Muller, 2009) of a NASA-VICAR-based pipeline developed by DLR (German Aerospace Centre) and JPL (Jet Propulsion Laboratory) has been employed with image matching based on the Gotcha (Gruen-Otto-Chau) algorithm (Shin and Muller, 2012) with a specialised setup for the polar region. DTM products have been produced with more than twice the resolution $(50 \mathrm{~m} / \mathrm{pixel})$ of the gridded Mars Orbiter Laser Altimeter (MOLA) 512 pixels/degree $(112 \mathrm{~m} /$ pixel) over the South Polar Residual Cap (SPRC) and the Mars South Polar region $\left(82^{\circ}-90^{\circ} \mathrm{S}\right)$ in MOLA and areoid reference. The accuracy of the HRSC orbital DTMs are compared against a MOLA reference with good results. HRSC orthorectified strip images from $12.5-50 \mathrm{~m}$ have also been produced from the base DTMs and these have been processed into a $12.5 \mathrm{~m}$ mosaic. HRSC strip products are currently being assessed as base images for automatic co-registration of thousands of high-resolution images, making them geometrically consistent with the surface conditions imaged by HRSC. In some cases, CTX DTMs have been automatically produced and co-registered to the HRSC image strips and these, in turn, are being employed for automated co-registration of higher-resolution images.
\end{abstract}

Keywords: Mars; SPRC; DTM; Mars South Pole; HRSC

\footnotetext{
${ }^{*}$ Corresponding author

Email addresses: alfiah.putri.15@ucl.ac.uk (Alfiah Rizky Diana Putri), p.sidiropoulos@ucl.ac.uk (Panagiotis Sidiropoulos), j.muller@ucl.ac.uk (Jan-Peter Muller), sebastian.walter@fu-berlin.de (Sebastian H. G. Walter), gregory.michael@fu-berlin.de (Greg G. Michael)
} 


\section{Introduction}

The Mars south polar region has generated research interest because of its rapidly changing appearance. Telescopic observations of Mars on Earth dates back to Huygens (1672) (see (Sheehan, 1996)), until the more recent James, et. al. James (1979) and from Hubble Space telescope (Cantor et al., 1998, James et al., 1979). Herschel Herschel (1784) (from (John Louis Emil, 2013), see also (Sheehan, 1996)) made notable early observations of the south polar cap. After the launch of the Viking and Mariner 9 spacecraft, the observations extended to quantifying the growth and recession of the ice caps (James, 1979 Piqueux et al., 2015: Calvin et al., 2017) and the dark ring shown around the South Pole (Geissler, 2005). With the launch of other spacecraft instruments, more information has been obtained about the SPRC (South Polar Residual Cap) (Thomas et al., 2000, 2005, 2009, 2016, Byrne and Ingersoll, 2003), finer features such as pits and troughs(Thomas et al. | 2009) and araneiform (spiderlike) terrain in the cryptic region (Piqueux et al. 2003, Kieffer, 2007) in MOCNA images, down to meter-and sub-meter-scale pitting and cracking in the SPRC (Buhler et al., 2017) observed in HiRISE images.

A Digital Terrain Model (DTM) is a gridded elevation model of the terrain surface, where each point has a regular position in latitude and longitude as well as an associated elevation value. For this research, single-instrument and multi-instrument images are used, automatically co-registered to base images and orthorectified to the same base DTM to widen spatial and temporal coverage of high-resolution images over the South Pole in order to obtain the most accurate representation of the surface. The only currently available DTM for the South Pole is from the Mars Global Surveyor laser altimeter MOLA with a resolution of $463 \mathrm{~m} /$ pixel on average globally and 512pixel/ degree over the polar region. MOLA produces pulse footprints diameter of $170 \mathrm{~m}$ on the Martian surface (Smith et al., 2001a: Poole et al., 2014). Given the differences of resolution from MOLA to HiRISE, the finest resolution camera of Mars currently reaches 400 times the mean resolution of the MOLA DTM. The MOLA DTM is therefore not ideally suited for performing terrain correction of higher resolution images through orthorectification. Although 18m CTX DTMs are being produced(Tao and Muller, 2017), their coverage in polar regions is poor. Serendipitous HiRISE stereo-pairs for making DTMs are even sparser.

This research is motivated by the lack of such high-resolution DTMs and corresponding orthorectified images (ORIs), which hinders the scientific analysis of this area. Research on the South Pole so far has been done using images from MOC-NA, CTX, and HiRISE, but hardly at all with HRSC. Because of the lack of high-resolution DTMs, these high-resolution images have mostly been orthorectified using the MOLA DTM. Even the currently available HRSC images are ortho-rectified to a $5 \mathrm{~km} \times 5 \mathrm{~km}$ MOLA DTM in most cases. For change detection, images are mostly co-registered to one another by hand (Buhler et al. 2017) so although the images may be properly co-registered between a subset of images, they are not all co-registered to a common baseline, which will present problems for further research. 
Another motivation for the creation of high resolution DTMs, especially with regards to polar research, is to fill the gap in the MOLA data product between $87^{\circ}-90^{\circ} \mathrm{S}$. This gap results from the non-polar orbit of MOLA's host spacecraft, the NASA Mars Global Surveyor. This gap is currently interpolated to produce a rasterised MOLA MEGDR South Polar DTM. The SPRC also lies within this gap, so it is critical for research over the SPRC to utilise a more complete DTM. One of the problems with the South Polar region is that the surface changes rapidly, resulting in problems in image matching. Another problem is the changeable weather conditions in certain seasons, which often reduce the input image quality due to dust storms, cloud and $\mathrm{CO}_{2}$ frosts, resulting in poor visibility of the surface.

The objective of this paper is to describe the processes involved in the creation and application of high-resolution DTMs from HRSC over the South Polar region. These DTMs are subsequently shown to be useful as base DTMs for generating orthorectified HRSC images. The orthorectified images can be subsequently used to produce higher resolution co-registered DTMs from repeat-pass CTX images, which themselves can subsequently be used to co-register finer DTMs and ORIs from HiRISE stereo-pairs. This method increases the accuracy of the resulting CTX and HiRISE DTMs compared to using MOLA DTM as the base DTM using the USGS ISIS+SOCET stereo photogrametric methods (Kirk et al., 2008). Given the orthorectified HRSC images, other high-resolution images can be co-registered to the same global reference as well as orthorectified to a common DTM. This is not only helpful for research using single-instrument images, but also for research using multiple-instrument images. This technique has already been demonstrated in other areas of Mars where high-resolution DTMs derived from HRSC data have been produced (Sidiropoulos and Muller. 2018, Sidiropoulos et al., 2018).

This paper is structured as follows. In Section 2 the requirements for a Mars DTM are discussed as well as the selection criteria utilised for the data used to produce the high-resolution South Polar DTM. Section 3 discusses the DTM production methods for Mars, planetary, and earth data and the DTM processing chain used in this paper. Section 4 shows the results of our DTM production. Section 5 shows the validation and assessment using the MEGDR MOLA grid and nadir images orthorectified to MOLA DTM, how the DTM and ORI products can be used for estimating changes in the ice cap volume and observing surface changes, discussion of the produced DTMs as well as on-going coregistration work with the produced HRSC ORIs. The last section concludes and discusses future work.

\section{Mars Images and Available Mars South Polar DTMs}

Mars has been observed intensively, especially at visible wavelengths over the last 40 years. High-resolution observations of Mars (defined here as IFoV (Instantaneous Field of View) $\leq 100 \mathrm{~m}$ ), were started by Viking Orbiter 1 and Viking Orbiter 2 with the Visual Imaging Subsystem (Soffen and Snyder, 1976), which were operational from 1976-1980. A global coverage of imagery finer than 
$1 \mathrm{~km} /$ pixel was obtained and the planet's first global mosaic was produced by USGS at $231 \mathrm{~m} /$ pixel, comprising some 4600 images out of the total of 47,000 images taken by both orbiters. Serendipitous stereo was acquired (Blasius et al. 1980) and automated stereo photogrammetric mapping has also been previously demonstrated (Thornhill et al., 1993).

The Mars Orbiter Camera-Narrow Angle (MOC-NA) on board the NASA Mars Global Surveyor (MGS) took pictures with resolutions ranging between 1.5-12m/pixel. Operating between 1997-2006 (Mars Year (MY)23-MY28), it imaged $5.45 \%$ of the surface of Mars at $12 \mathrm{~m} / \mathrm{pixel}$, and began the acquisition of stereo coverage of Mars, resulting in a $0.32 \%$ stereo coverage (Malin et al. 2010 ).

Better surface coverage was obtained by the Thermal Emission Imaging System (THEMIS-VIS) onboard the NASA Mars Odyssey at $18 \mathrm{~m} /$ pixel (Christensen et al., 2004). More than $82.01 \%$ of Mars surface has been covered by this instrument since 2001, and it is still in operation in early 2018. The High Resolution Stereo Camera (HRSC) onboard of the ESA Mars Express started its operation in 2003 (MY26) and is still working until the present-day (Gwinner et al. 2016). The NASA Mars Reconnaisance Orbiter was launched in 2006 (MY28) with two visible instruments on board, the Context Camera (CTX) and the High Resolution Imaging Science Experiment (HiRISE). CTX has a nominal resolution of $6 \mathrm{~m} /$ pixel and provides context to the instrument with the highest resolution so far, HiRISE with $0.25-0.5 \mathrm{~m} /$ pixel. Both are still working as this paper is written, covering more than $82 \%$ of Mars for CTX and $2.40 \%$ of Mars for HiRISE, with $28.21 \%$ CTX DTM coverage(Tao et al., 2018) and $0.183 \%$ stereo HiRISE coverage ((Tao et al., 2018), also see (Becker et al. 2015: Mattson et al., 2011)). Recently the ExoMars Trace Gas Orbiter has been launched, with Colour and Stereo Surface Imaging System (CaSSIS) which is taking 2-4.5m/pixel stereo images of Mars since May 2018(Thomas et al., 2014).

\section{1. $M O L A$}

The Mars Orbital Laser Altimeter instrument is onboard the Mars Global Surveyor (MGS) launched on 7 November 1996 (MY23) and failed on 2 November 2006. With an along-track sampling frequency of $10 \mathrm{~Hz}$, this $1064 \mathrm{~nm}$ laser altimeter, MOLA collected altimetry data of Mars with a pulse footprint diameter of 170m every 300m until 30 June 2001 (MY25) (Seidelmann et al., 2002). The MOLA Precision Experiment Data Records (PEDRs) are generated from raw altimetry profile data with precision orbit corrections applied.

From these "points", three sets of rasterised grids were produced by interpolating the values obtained by MOLA, called the MOLA MEGDR (Mission Experiment Gridded Data Records). MOLA MEGDR consist of a global Martian DTM, a South Polar DTM up to a latitude of $-70^{\circ} \mathrm{S}$ and a North Polar DTM down to a latitude of $70^{\circ} \mathrm{N}$. Global Martian DTMs are available with resolutions of 4,16,32, 64 and 128 pixels/ degree (which are about to 15, 3.7, $1.85,0.9$, and $0.463 \mathrm{~km} /$ pixel respectively) for global data. Because of its global coverage, its consistency from crossover analysis and its accuracy, MOLA data 
is treated as the principal areocentric reference body for Mars (Duxbury et al. 2002).

In regards to the South Polar DTMs, although the resolution of the global DTM is too coarse for our intended goal, because of its polar orbit, the MOLA point density is higher near the poles. A consequence of the near-polar orbit is that the resolution of the raster product can be increased up to 512 pixels/ degree. However, this is at the expense of a large gap from $87^{\circ}-90^{\circ}$ where no laser tracks are available. This is a problem for any research involving images for the production of higher resolution DTM over this area.

\section{2. $H R S C$}

The ESA-DLR High Resolution Stereo Camera (Neukum and Jaumann 2004) has been active in orbit since January 2003 (MY27), and is still acquiring pictures of Mars whilst this paper is written. HRSC has reached global coverage of $97 \%$ for resolutions of $100 \mathrm{~m} /$ pixel and higher and $70 \%$ at $10-20 \mathrm{~m} /$ pixel (Gwinner et al., 2016). HRSC obtains images in resolution ranging between $200 \mathrm{~m} /$ pixel at its coarsest up to $12.5 \mathrm{~m} /$ pixel at its finest, athough HRSC data at lower resolution are also acquired of limbs and the poles. This difference in resolution is due to its elliptical orbit due to the need to accommodate non planetary surface instruments as well as limited onboard storage for acquiring all views at full resolution for each data acquisition.

HRSC is a pushbroom camera with 9 different optical components at 9 different angles ranging up to $\pm 18.9^{\circ}$ : one nadir channel, two stereo channel, four-band colour channels (Red, Green, Blue, and Near Infra-Red) and two photometry channels. Another (frame array) camera, the Super Resolution Camera (SRC) has 2-3 times the resolution of the nadir camera. With this setup, HRSC is currently the only stereo camera orbiting Mars (CaSSIS has yet to start obtaining stereo images at the time of writing). This uniqueness combined with its coverage ensures that close to $100 \%$ of the surface of Mars is available in stereo with temporal effects being almost negligible $(\leq 120 \mathrm{~s})$.

Although the coverage of HRSC images orthorectified to a MOLA DTM over the South Pole appears satisfactory, the polar gap and the large difference in resolution between HRSC images (up to $12.5 \mathrm{~m} / \mathrm{pixel}$ ) and the MOLA MEGDR resolution of $112 \mathrm{~m} /$ pixel results in poor orthorectification in this region. However, one of the major difficulties to ensure that HRSC strip products will lie on top of each other is that accurate spacecraft location and viewing orientation (which is referred as exterior orientation in this paper) is required for each strip, preferably from a bundle block adjustment, but in practice this is not feasible with the resources available. There were 14 DTMs over the area of interest available on the HRSC database at the ESA-PSA. However, their coverage is limited and their quality variable (see Figure 1). After searching through the metadata for HRSC south polar images for those acquired during a similar season (solar longitude, $\mathrm{L}_{\mathrm{s}}$ ), similar nadir resolution $(12.5 \mathrm{~m}, 25 \mathrm{~m}$, or $50 \mathrm{~m}$ ) strips, and examining each image for cloud or dust cloud coverage visually as well as by studying the MARCI image sequences kindly provided by Wendy Calvin (private communication, 2016) it was determined that there were sufficient numbers 


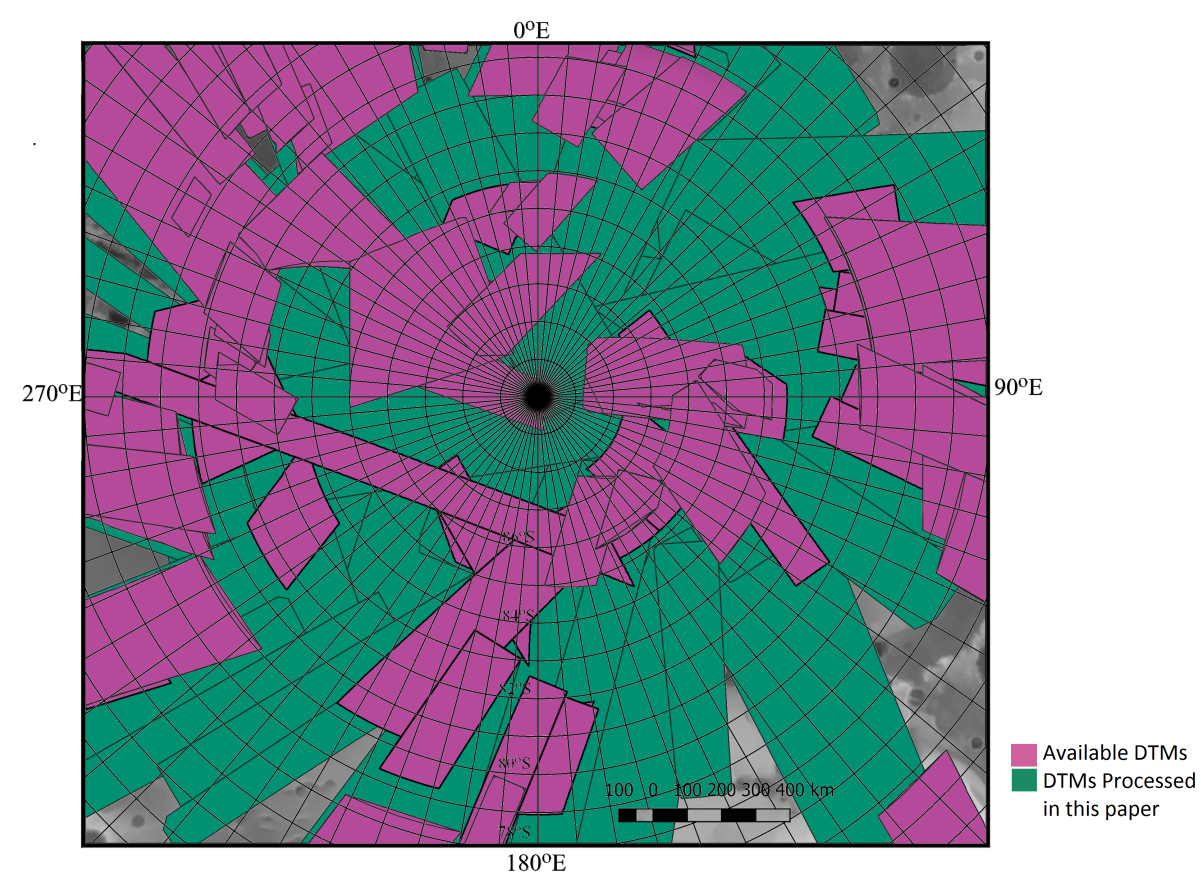

Figure 1: Currently available DTMs over the SPRC and south polar region

of overlapping HRSC images which could be photogrammetrically processed to cover $100 \%$ over the area.

Based on the spatial coverage, it was decided that producing DTMs for the South Polar region was feasible at $50 \mathrm{~m}$, which is around twice the best MOLA DTM resolution. The HRSC DTMs also completely cover the gap below $87^{\circ}$ ) $\mathrm{S}$ and allow the production of a set of base orthorectified images. The usual way to look for stereo pair images is to look for overlapping images with similar criteria such as incidence angle, solar azimuth, etc. (Becker et al., 2015), and not necessarily to give much attention to solar longitude values. These usual criteria to look for stereo pairs is not necessarily suited to the polar region, because of the existence of surface changes caused by seasonal processes causing different surface appearances for two stereo pairs. In this region, similarity in solar longitude increases the success rate for stereo matching. The changes signified by solar longitude values are obvious and cause a real problem for stereo matching if the features between two stereo images don't match because of the surface changes in the time interval between the dates they were captured. As all 9 HRSC CCD images are taken near-simultaneously (within 2 minutes), problems caused by these possible changes can be minimised. The last benefit of using HRSC and stereo imagery compared to using altimeters such as MOLA is that the CCD images are taken at almost exactly the same time and can be more readily used for matching. This brightness information can be used to 
improve point sampling such as used in block adjustment to update exterior orientation.

A topographic datum of Mars has been defined based on a gravity model obtained by the Mars Global Surveyor(Lemoine et al., 2001). On Mars, there are several reference height frames for DTMs including: the MOLA Sphere defined by the MOLA mission as a sphere with $3396 \mathrm{~km}$ radius; an areoid surface, defined by the zero-elevation surface described previously. The Viking MDTM is available also on an older datum, but both MOLA MEGDR DTMs and HRSC DTMs are available in both reference systems. We also provide south polar DTMs in both reference systems.

\section{Digital Terrain Model Production Pipeline}

HRSC DTMs of the surface of Mars are produced exploiting its capabilities as a stereo photogrammetric camera system. The method used for producing HRSC DTMs by the DLR team is based on the recently published open-source NASA VICAR software, with specific proprietary executable software developed at DLR for producing DTMs from HRSC images (DLR-VICAR) (Scholten et al. 2005). Using MOLA data as control for HRSC exterior orientation, the DTMs produced can obtain vertical accuracies averaged at 12m (Gwinner et al. 2010, 2016). Other papers have also mentioned a method that has been previously discussed for HRSC by producing a DTM using the commercial SOCET-SETR which uses a generic camera model and combining this with ISIS (Integrated Software for Imagers and Spectrometers) (Kirk et al. 2002, 2008) Improvements were made based on the original VICAR-based DTM production pipeline such as Kim and Muller (Kim and Muller, 2009) who produced an in-house method by modifying the original DLR-VICAR based pipeline to use the Gotcha (GruenOtto-Chau) algorithm (Shin and Muller, 2012) for densifying the DTM up to 30m grid-spacing (Heipke et al., 2007). The NASA Ames Stereo pipeline can also be employed to generate HRSC DTMs if suitable bundle adjusted and updated SPICE kernels are available (R. Beyer, private communication, 2018).

The method that was employed to produce the HRSC DTMs is described in Kim and Muller (2009). It is based on a DLR-VICAR pipeline which was briefly discussed as one of the DTM Production Methods in the previous paragraph. This is part of a complex PERL script to allow fully automated DTM processing (Stephan van Gasselt, private communication, 2005). Inputs for this pipeline are three Level 2 HRSC images, which means images that have been radiometrically calibrated to radiance or reflectance but are still in the original instrumentbased geometry. These three level 2 HRSC images are stereo triplet views from nadir, stereo angle 1 , and stereo angle $2\left( \pm 18.9^{\circ}\right)$. Other information needed to produce HRSC DTMs with this method are: a base DTM, which in this work is the Mars South Polar MOLA MEGDR, updated exterior orientation files (A. Dumke, private communication, 2016) obtained by bundle adjusting multiple channels from each orbit with MOLA data (Bostelmann and Heipke, 2016, Heipke et al., 2007), and geometric calibration data. 
Initially, images are orthorectified from Level 2 HRSC images (nadir, stereo angle 1, and stereo angle 2) using the DLR-VICAR orthorectification programme (Gwinner et al. 2009) annd a gridded version of MOLA heights (see next paragraph). Area-based matching is then performed on image patches from image pairs extracted from the three stereo images. Instead of using the DLR-VICAR programme to match conjugate points to nadir iteratively, each bilinearly sampled image is matched from the lowest resolution up to the highest using discrete pixel position approximation by a cross-correlation algorithm. Area based matching in this modification is performed using a region-growing variant of the Gruen Adaptive Least Square Correlation (ALSC) matcher within the Gotcha (Gruen-Otto-Chau) algorithm (Gruen, 1985, Otto and Chau, 1989 , Shin and Muller, 2012). For each match, a variance and co-variance matrix is defined and a maximum eigenvalue is calculated as a precision value. Iteration is performed to calculate the lowest possible maximum eigenvalue based on different matching window sizes, from larger to smaller. In this paper we use a threshold of 5 iterations to find the lowest possible maximum eigenvalue. Maximum grey level differences are also calculated as metrics (Kim et al., 2013). Pairwise matching results are merged and 3D points are computed using a least squares forward intersection. Blunders are reduced by assigning a threshold for intersection accuracy. More accurate subpixel retrievals are obtained by interpolation, and a final DTM is produced by kriging grid point interpolation. The overall production system is shown in Figure 2.

The processing is done in sections by dividing orbital strips (which can be up to 100,000 lines in length) into segments, each varying from 5,000-10,000 lines, and then merging them together afterwards using the DLR mosaicking software (Scholten et al., 2005) without grey-level adjustment and without elimination of duplicated values. Disparity between the images is set between the limits of \pm 5 pixels, and for Gotcha the minimum correlation is set to 0.6 and maximum eigenvalue is set to 600 . Artefacts caused by stereo matching over cliff edges (signified by very high difference to MOLA values compared to other data) are cropped afterwards and not used for calculation of the end products. To reduce the disparity range, MOLA DTMs are employed to orthorectify the imagery. Usually this is performed using a $5 \mathrm{~km} /$ pixel DTM. Instead here the 512 pixels/degree $(112 \mathrm{~m} /$ pixel) MOLA DTM is employed.

DTMs are produced with a grid spacing of $50 \mathrm{~m} /$ pixel from the original image resolutions, which range between $12.5-50 \mathrm{~m} /$ pixel. Orthorectified images are produced by orthorectifying Level 2 images used as inputs to the HRSC DTM produced at their original resolution of either $12.5,25$, or $50 \mathrm{~m} /$ pixel using a polar stereographic projection with the same projection parameters of $90^{\circ} \mathrm{S}$ and $0^{\circ} \mathrm{E}$ as centres of projection. This global projection is chosen instead of maximising matching by projecting images into their central longitude and latitude to obtain a similar reference so that we can produce a global map and mosaics from the product. A significant improvement was obtained by exploiting the Mars MEGDR (available from $87^{\circ} \mathrm{S}$ down to approximately $75^{\circ} \mathrm{S}$ ) in polar stereographic projection at the highest resolution (512 pixels/ degree) as the base DTM. This raster image has the advantage of obtaining higher accuracy 


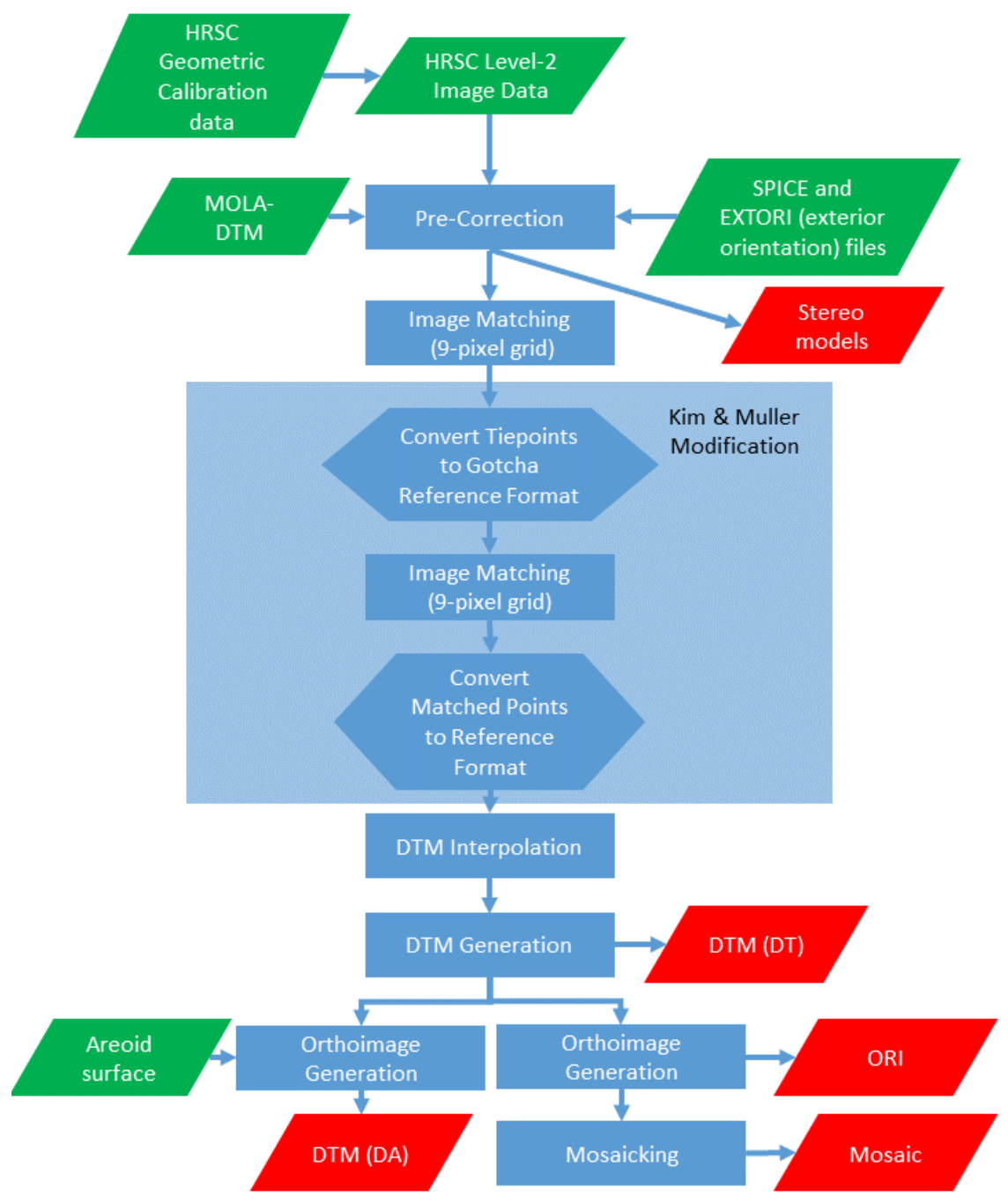

Figure 2: Schematic of modified DTM production algorithm based on DLR-VICAR (Kim and Muller 2009 
in the polar region, instead of using a global DTM in Plate Carée cylindrical projection, where MOLA counts per pixel are high for the higher latitudes, both resulting in less accuracy and unavailability to process images with poles. The areoid version of products are also produced by differencing DTM products with the Mars geopotential surface. Striping effects sometimes occur in areoid products because of the limited values of areoid surface stretched to $50 \mathrm{~m} /$ pixel and integration of values to $16 \mathrm{bit}$ integer. These have been fixed using the floating-points method discussed in Section 4

\section{Results}

In this paper, we analyse the new high-resolution DTMs generated from HRSC images over the South Pole. We selected 33 polar orbits based on their MY and their coverage (see coverage map in Figure 1). Emphasis is given to images from Mars Year 27 (23 strips, 69.7\%) with 8 other strips from MY28 (24.24\%) and 2 strips from MY29 to fill gaps. The detailedata acquisition information is presented in Table 1 .

The 33 DTMs cover almost the whole of the area between latitude of $82^{\circ} \mathrm{S}-$ $90^{\circ} \mathrm{S}$. There was a bundle adjustment failure for orbit H2153_0000, which had no other HRSC images overlapping this area. A DTM has been produced over this area, but we expect the accuracy to be lower because of the use of the predicted exterior orientations used to produce this DTM instead of the bundle-adjusted exterior orientation data. The elevations of the DTMs produced in this paper are given with height referenced to the MOLA sphere (DT extension in HRSC definitions) as well as areoid products (DA in HRSC definitions) which have been produced by subtracting the height from the difference from the South Polar MOLA MEGDR radius and MOLA MEGDR areoid heights. The small range of the areoid heights resulting in stripe-like patterns when the resulting areoid products was interpolated to integer. This was solved by using floating-point values for intermediate steps and adding a filter for $87^{\circ} \mathrm{S}-90^{\circ} \mathrm{S}$ where the striping result could still be observed.The coverage of the resultant DTMs products is illustrated in Figure 3. This DTM could then be mosaiced with the existing MOLA $112 \mathrm{~m} /$ pixel DTMs to fill any remaining few grid-points where there were gaps. The resolution of the MOLA DTM in the area without HRSC DTMs data gets upsampled to HRSC DTMs resolution $(50 \mathrm{~m} / \mathrm{pixel})$

\section{Discussion}

\subsection{DTM and Vertical Accuracy Analysis}

An accuracy assessment was performed by comparing the HSRC height results for each HRSC image strip against the equivalent MOLA height by downsampling the DTM products $(50 \mathrm{~m} / \mathrm{pixel})$ to the MOLA South Polar MEGDR resolution and subtracting the values from the MOLA MEGDR. From the relative accuracy map, we can see that differences with MOLA DTM are generally positive in the north-west direction and negative in the south-east direction. 
Table 1: Information for each HRSC DTM strip produced

\begin{tabular}{lclcl}
\hline Product ID & Nadir image Scale $(\mathrm{m} / \mathrm{pixel})$ & Solar Longitude & Mars Year & Date \\
\hline H2153_0000 & 50 & 289.93 & 27 & $17-09-2005$ \\
H2155_0000 & 50 & 290.27 & 27 & $18-09-2005$ \\
H2160_0000 & 25 & 291.13 & 27 & $19-09-2005$ \\
H2162_0000 & 25 & 291.47 & 27 & $20-09-2005$ \\
H2163_0000 & 25 & 291.64 & 27 & $20-09-2005$ \\
H2165_0000 & 25 & 291.98 & 27 & $20-09-2005$ \\
H2167_0000 & 25 & 292.32 & 27 & $21-09-2005$ \\
H2179_0000 & 25 & 294.36 & 27 & $24-09-2005$ \\
H2181_0000 & 25 & 294.70 & 27 & $25-09-2005$ \\
H2183_0000 & 25 & 295.04 & 27 & $25-09-2005$ \\
H2189_0000 & 25 & 296.05 & 27 & $27-09-2005$ \\
H2197_0000 & 25 & 297.40 & 27 & $29-09-2005$ \\
H2286_0000 & 25 & 312.13 & 27 & $24-10-2005$ \\
H2287_0000 & 25 & 312.30 & 27 & $25-10-2005$ \\
H2288_0000 & 12.5 & 312.46 & 27 & $25-10-2005$ \\
H2318_0000 & 12.5 & 317.29 & 27 & $02-11-2005$ \\
H2340_0000 & 12.5 & 320.79 & 27 & $08-11-2005$ \\
H2348_0000 & 12.5 & 322.06 & 27 & $11-11-2005$ \\
H2362_0000 & 12.5 & 324.26 & 27 & $15-11-2005$ \\
H2372_0000 & 12.5 & 325.82 & 27 & $17-11-2005$ \\
H2428_0000 & 12.5 & 334.45 & 27 & $03-12-2005$ \\
H2454_0000 & 12.5 & 338.37 & 27 & $10-12-2005$ \\
H2498_0000 & 12.5 & 344.90 & 27 & $23-12-2005$ \\
H4144_0000 & 50 & 208.88 & 28 & $29-03-2007$ \\
H4162_0000 & 50 & 211.95 & 28 & $03-04-2007$ \\
H4369_0000 & 12.5 & 248.22 & 28 & $31-05-2007$ \\
H4574_0000 & 12.5 & 284.34 & 28 & $27-07-2007$ \\
H4685_0000 & 12.5 & 303.18 & 28 & $27-08-2007$ \\
H4696_0000 & 12.5 & 305.00 & $30-08-2007$ \\
H4837_0000 & 25 & 327.62 & $09-10-2007$ \\
H4917_0009 & 25 & 249.80 & $31-10-2007$ \\
H6794_0000 & 12.5 & 270.10 & $18-04-2009$ \\
H6908_0000 & 25 & & $21-05-2009$ \\
\hline & & 28 &
\end{tabular}




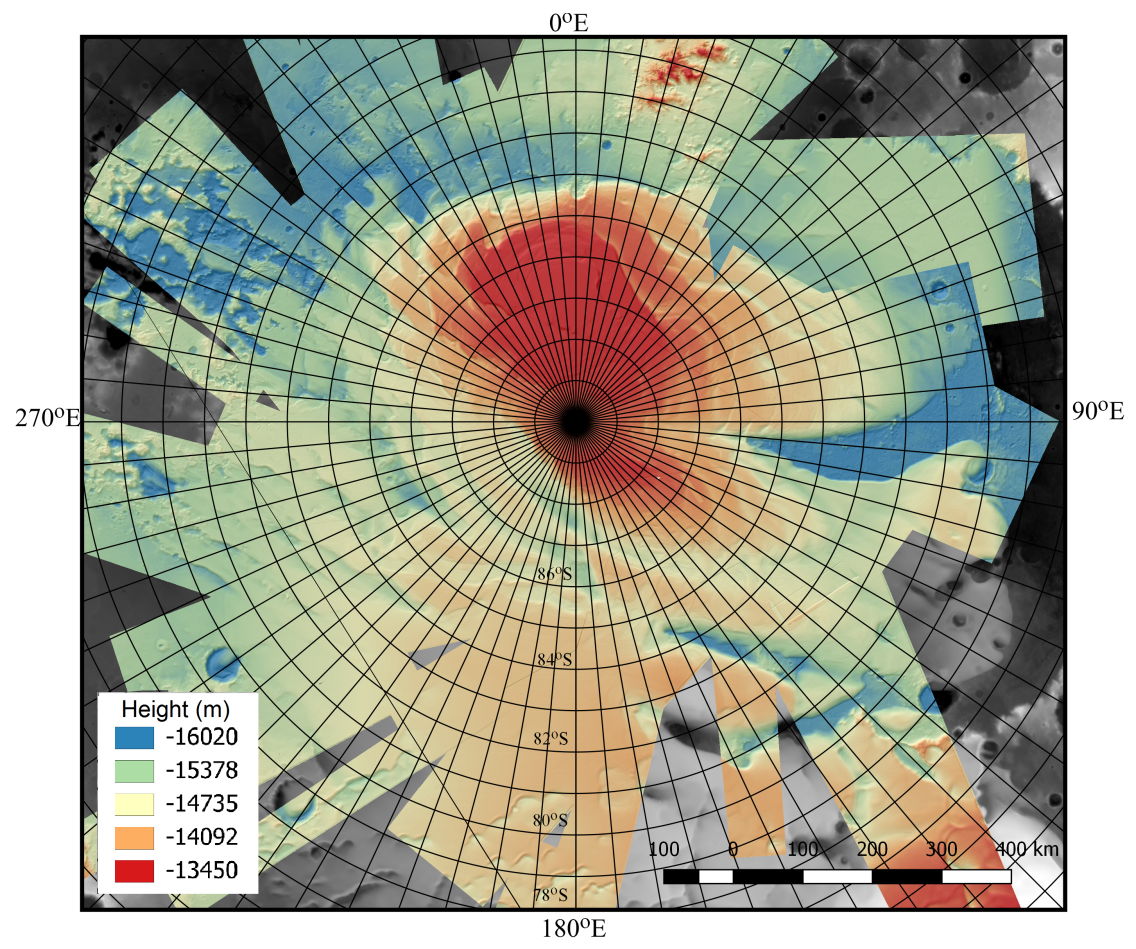

Figure 3: HRSC DTMs produced for the SPRC. Height information is measured from MOLA DTM sphere. 


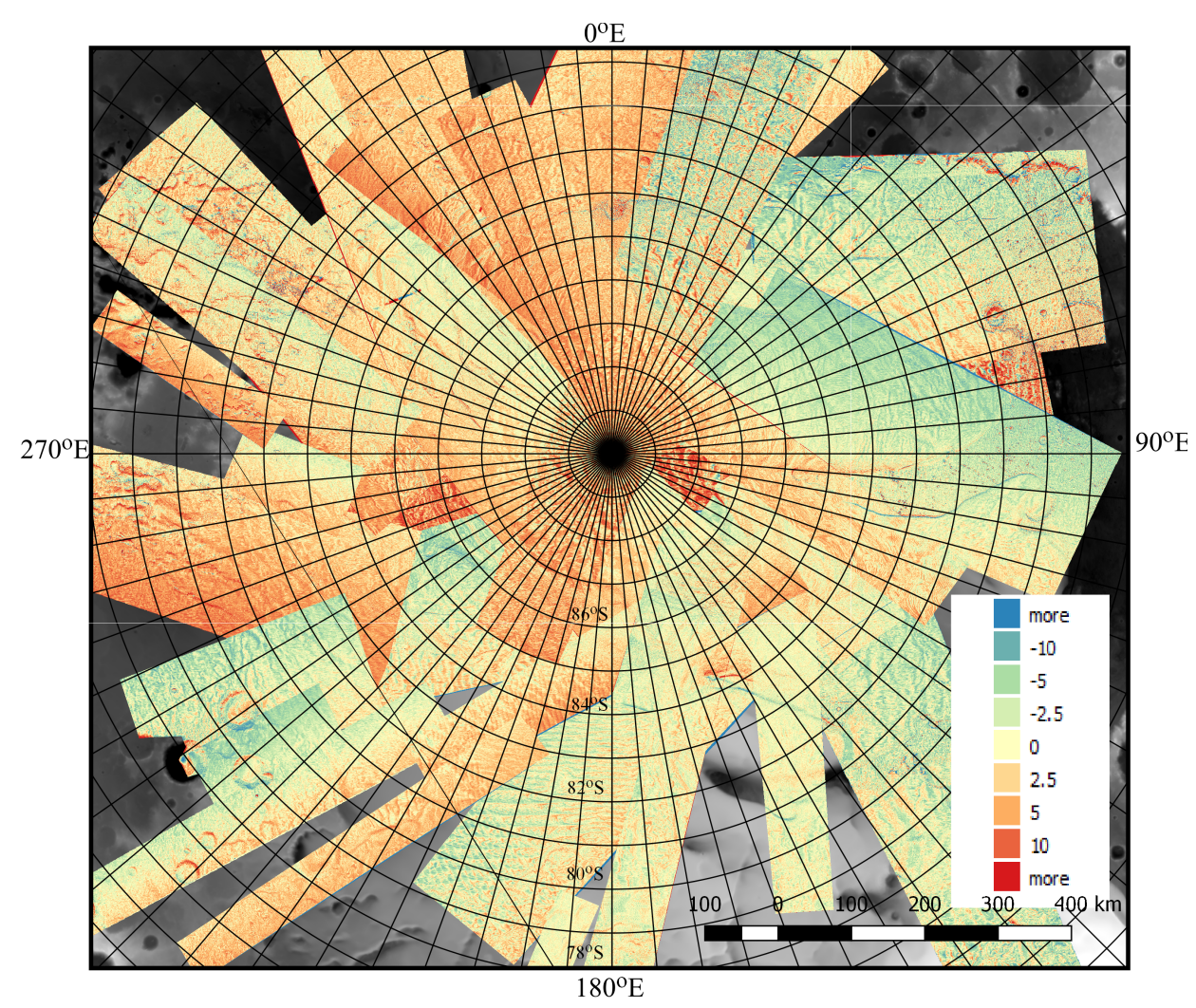

Figure 4: Height accuracy assessment for HRSC DTM strips to MOLA MEGDR. The figure shows differences to MOLA MEGDR in metres. 


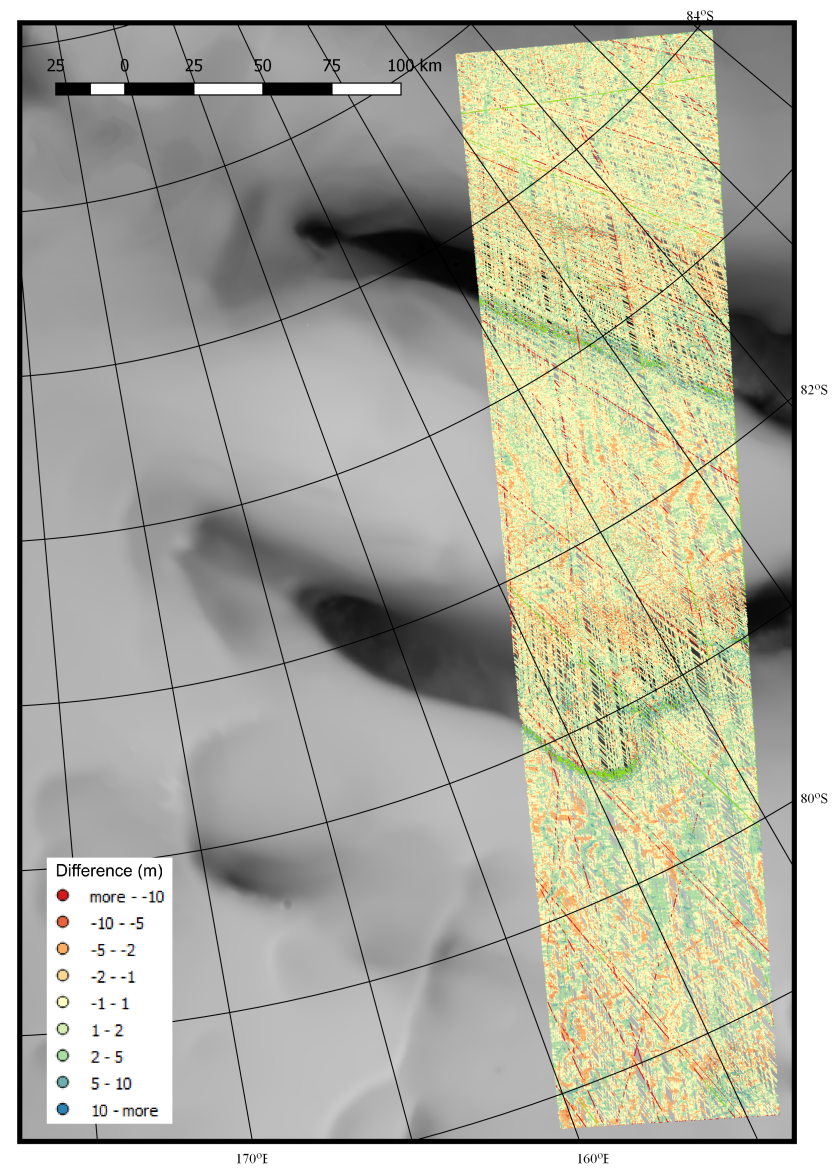

Figure 5: Height accuracy assessment for HRSC DTM strips to MOLA PEDR points (the example is H2348_0000 (11-11-2005, MY27, L 322.06$)$ ). The figure shows differences to the MOLA PEDR points in metres. 
An illustration of this difference can be seen in Figure (4) and the statistics for each strip is presented in Table 2, showing the mean of the differences between HRSC points to MOLA points, with $\sigma$ as the standard deviation of the differences, RMSE as the root mean square error of the differences, |Mean| as the overall mean of the absolute values of the differences, max and min as maximum and minimum height difference excluding outliers with range as the difference between the two values, Med as the median value, and counts as the number of MOLA points for each strip. Using this method the overall mean difference (bias) with MOLA MEGDR is $1.08 \mathrm{~m}$ with a root mean square error of $7.29 \mathrm{~m}$ and a mean absolute difference of $3.21 \mathrm{~m}$. This value is comparable with the results calculated for the currently available HRSC DTMs overlapping the MOLA South Polar MEGDR. The new DTMs were compared with the currently available DTMs (H2364_0000, H4526_0000, H4547_0000). Three of these HRSC products are all located around a similar area $\left(86^{\circ} \mathrm{S}-88.5^{\circ} \mathrm{S}, 291^{\circ} \mathrm{W}-24^{\circ} \mathrm{E}\right)$ with a mean difference to MOLA MEGDR more than $12 \mathrm{~m}$ and a standard deviation higher than $40 \mathrm{~m}$. These DTMs are complemented by DTMs overlapping the same area produced in this paper (H2181_0000, H2189_0000, H4837_0000, H6908_0000), with a mean difference between $0.29 \mathrm{~m}-4.37 \mathrm{~m}$ and a standard deviation of $3.07 \mathrm{~m}-10.66 \mathrm{~m}$. The mean differences between overlapping DTMs are less than the RMS errors in the data products (Table 2), which indicates that there are no vertical changes detectable in the HRSC DTMs over this region between MY 27 and MY 29.

The method used in this paper also worked well in both rugged areas (signified by high elevation deviations) and smoother plains (vice versa), and there are no significant differences between these values for the three most rugged strips (H2160_0000, H2318_0000, and H4369_0000) and the three smoothest strips (H2155_0000, H2340_0000, H6908_0000). A consistent grid spacing of $50 \mathrm{~m} /$ pixel for all DTMs is used to prevent sacrificing information for higher resolution input images and for producing geospatial analysis product (mosaic). From the vertical accuracy assessment, we can also assert that producing 50 $\mathrm{m} /$ pixel DTMs from $50 \mathrm{~m} /$ pixel nadir images is acceptable for the south polar region because of the resolution of the base DTM $(112 \mathrm{~m} /$ pixel $)$ which is about twice the resolution of the images and the sub-pixel Gotcha image matcher used to produce sub-pixel accuracy. The actual finest grid-spacing depends on many different factors, including the texture and the Signal to Noise Ratio. These fine details are not considered in this paper. Height assessment with MOLA MEGDR also gives satisfactory results for orbit H2153_0000 compared to other strips. Based on the resulting DTMs, the mean differences with MOLA MEGDR for each nadir resolution group are $0.89 \mathrm{~m}(12.5 \mathrm{~m} / \mathrm{pixel}), 1.98 \mathrm{~m}(25 \mathrm{~m} / \mathrm{pixel})$, and $-1.41 \mathrm{~m}(50 \mathrm{~m} / \mathrm{pixel})$.

Analysis has also been performed for each strip using the original MOLA PEDR values (Smith et al., 1999). The MOLA PEDR points are extracted for each orbit and DTMs are discretely sampled to compare the height to MOLA PEDR points. Comparison has been done for HRSC DTM heights and MOLA heights. A mean difference is calculated for each orbit by subtracting sampled points from the MOLA PEDR points. Validation is done to assess DTM quality 
Table 2: Vertical Difference Assessment of Processed Products to MOLA MEGDRs and PE-

\begin{tabular}{|c|c|c|c|c|c|c|c|c|c|c|c|c|c|}
\hline \multirow[b]{2}{*}{ Product ID } & \multicolumn{8}{|c|}{ Assessment to MOLA MEGDR } & \multicolumn{5}{|c|}{ Assessment to MOLA PEDR Points } \\
\hline & Mean & $\sigma$ & RMSE & Mean & Max & Min & Range & Med & Mean & $\sigma$ & RMSE & Mean & Counts \\
\hline H2153_0000 & -1.34 & 5.68 & 5.83 & 2.88 & 125 & -360 & 485 & -1 & 0.44 & 23.44 & 23.45 & 5.04 & 3610642 \\
\hline H2155_0000 & -0.32 & 4.76 & 4.77 & 0.83 & 138 & -364 & 502 & 0 & 1.01 & & 15.26 & 5.22 & \\
\hline H2160_0000 & 4.81 & 3.84 & 6.18 & 5.11 & 102 & -567 & 669 & 5 & & & 18.27 & 6.46 & 046 \\
\hline H2162_0000 & -0.32 & 4.76 & 4.77 & 0.83 & 138 & -364 & 502 & 0 & -2.50 & & 11.03 & 2.91 & 013 \\
\hline _0000 & 3.09 & 2.55 & 3.99 & 3.36 & 40 & -209 & 249 & 3 & -3.29 & & 15.65 & 4.92 & 991 \\
\hline _0000 & 0.84 & 0.35 & 0.9 & 0.84 & 188 & -91 & 279 & -2 & 6.26 & & 31.62 & 8.81 & 193 \\
\hline _0000 & 3.94 & 13.18 & 13.7 & 5.22 & 198 & -232 & 430 & 0 & -4.84 & & 30.42 & 7.81 & 2849 \\
\hline _0000 & -1.80 & 13. & & 3.36 & 44 & -1293 & & 0 & 0.74 & & 20.67 & 4.98 & \\
\hline _0000 & 2.02 & 10.66 & .83 & 2.15 & 205 & -281 & 4 & 0 & -5.17 & & 21.93 & 6.43 & 4230094 \\
\hline 000 & -4.15 & 10.03 & 10.71 & 4.22 & 207 & -448 & 55 & -4 & 4.04 & & 13.37 & 5.41 & 075 \\
\hline $\mathrm{H} 21$ & 1.72 & 7.09 & .27 & 2.45 & 213 & -179 & 92 & 1 & -3.73 & & 25.61 & 5.86 & \\
\hline 2197_0000 & -1.6 & 3.93 & 4.19 & 2.87 & 43 & -233 & 276 & -1 & 1.08 & & 14.18 & 4.20 & 759573 \\
\hline H2286 & 7.49 & 32.54 & 33.8 & 13.04 & 169 & -239 & 8 & 0 & -0.72 & & .77 & .04 & \\
\hline _0000 & -23.1 & 57.03 & 1.41 & 25.42 & 379 & -525 & 904 & -2 & 0.38 & 25.04 & 25.04 & 4.82 & 597345 \\
\hline 00 & 0.76 & 4.41 & 2 & 2.74 & 75 & -616 & 6 & 1 & -2.58 & & 35.33 & 7.8 & 102 \\
\hline $\mathrm{H} 23$ & -3.05 & 3.68 & 4.7 & 3.46 & 46 & -133 & 179 & -3 & 2.10 & & 18.55 & 5.48 & \\
\hline 0000 & 0.19 & 3.00 & 2.9 & 2.19 & 43 & -49 & 9 & 0 & 0.41 & & 24.93 & 4.86 & \\
\hline $\mathrm{H} 23$ & -0.36 & 3.26 & 3.28 & 1.07 & 134 & -130 & 264 & 0 & 0.04 & & 13.52 & 3.07 & 729 \\
\hline $\mathrm{H} 236$ & -3.05 & 3.68 & 4.75 & 3.46 & 46 & -133 & 179 & 3 & -2.43 & & 21.12 & 5.80 & 2167703 \\
\hline $\mathrm{H} 23$ & 3.78 & 24.42 & 24.78 & 4.93 & 270 & -159 & 429 & 1 & -3.55 & & 34.08 & 7.33 & 91 \\
\hline $\mathrm{H} 24$ & 2.14 & 7.37 & 7.69 & 3.81 & 24 & -106 & 1 & 3 & -2.45 & & 20.16 & 5.61 & 097 \\
\hline $\mathrm{H} 24$ & 0.00 & 1.63 & 1.6 & 1.14 & 36 & -73 & 109 & 0 & -0.91 & & 15.52 & 2.58 & 980 \\
\hline H249 & 0.03 & 2.70 & 2.64 & 0.33 & 124 & -1365 & 1489 & 0 & -2.18 & & 25.86 & 4.25 & 458 \\
\hline 000 & -1.30 & 7.37 & 7.49 & 3.98 & 268 & -930 & 1198 & -1 & 0.82 & & 14.76 & 5.24 & 2635904 \\
\hline H416 & -2.43 & 6.26 & 6.78 & 3.19 & 77 & -551 & 628 & -2 & 1.99 & 14.90 & 15.04 & 4.72 & 3996424 \\
\hline H43 & 1.3 & 4.06 & 4.25 & 2.44 & 109 & -75 & 184 & 1 & -2.48 & 20 & 20.54 & 4.33 & 371309 \\
\hline 000 & 3.72 & 10.17 & 10.84 & 5.54 & 57 & -175 & 232 & 4 & -3.65 & & 15.91 & 6.85 & \\
\hline H4685_0000 & -0.42 & 10.89 & 10.89 & 2.78 & 251 & -198 & 449 & -1 & -0.60 & 20.75 & 20.76 & 4.50 & 1137623 \\
\hline _0000 & 0.53 & 6.52 & & 2.32 & 209 & -1431 & 1640 & 0 & -2.34 & 22.65 & 22.78 & 4.74 & 1429417 \\
\hline H4837_0000 & 0.29 & 4.64 & 4.00 & 1.39 & 281 & -356 & 637 & 0 & -2.19 & 25.75 & 25.84 & 7.12 & 4022651 \\
\hline H4917_0009 & 5.90 & 7.66 & 9.61 & 7.27 & 154 & -314 & 468 & 6 & -7.72 & 18. & 19.00 & 10.25 & 6477732 \\
\hline H6794_0000 & 3.51 & 6.15 & 7.04 & 3.90 & 167 & -270 & 437 & 3 & -4.53 & 18.00 & 18.57 & 5.77 & 2183158 \\
\hline H6908_0000 & 4.37 & 3.07 & 5.34 & 4.54 & 51 & -218 & 269 & 4 & -5.27 & 21.87 & 22.50 & 6.36 & 3236488 \\
\hline
\end{tabular}

$t$ Difference is calculated in metres. Counts are number of MOLA points in an individual strip 


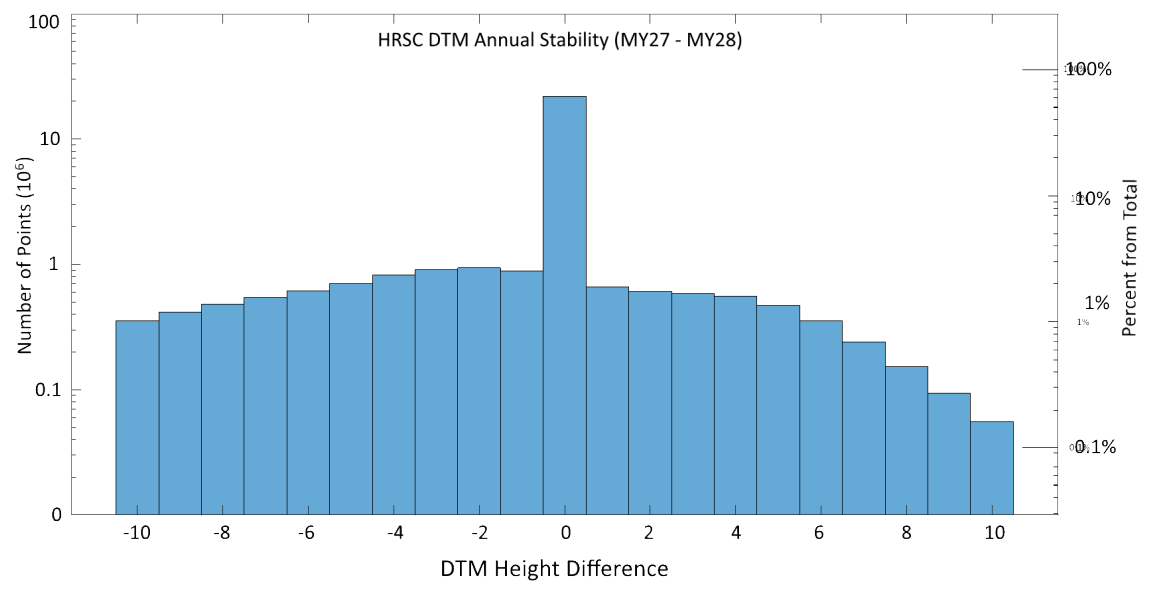

Figure 6: Annual stability testing, analysed by differencing heights between MY27-MY28, not including H2287_0000, both tails not shown for clarity. The graph is presented in logarithmic scale because the percentage of height difference of $\leq 1 \mathrm{~m}$ is far higher compared to other height differences

with MOLA PEDR results and results in a mean difference (bias) of $0.04 \mathrm{~m}$ difference and $13.53 \mathrm{~m}$ of root mean square error for DTMs with a resolution of $50 \mathrm{~m} /$ pixel. An example of the height difference map for these PEDR point is shown in Figure 5 .

From this assessment against PEDR points by separate strips shown in Table 2. we obtain a mean differences of $-2.20 \mathrm{~m}$ to PEDR points with $-9.48 \mathrm{~m}$ root mean square error and $6.12 \mathrm{~m}$ mean absolute difference. This mean difference is larger than the differences assessed using the MOLA MEGDR because of the likelihood that in the DTM production we use MOLA MEGDR heights which are gridded values of PEDR data as the base DTM.

\subsection{Annual and Seasonal Stability}

Annual and seasonal stability assessments are necessary to be performed for the South Polar images considering the annual and seasonal changes caused by the recession and growth of the seasonal $\mathrm{CO}_{2}$ ice cap (Smith et al. 2001b). To look at annual stability, we compare DTMs produced from overlapping strips from different years. As shown in Figure 6 is a histogram of height differences for DTM heights taken in PEDR point locations between MY27 and MY28 with both tails cropped and focused within $-10 \mathrm{~m}$ until $10 \mathrm{~m}$ because the percentage of values outside those numbers is very low. In this calculation we do not include H2287_0000 as statistically based on 2 its difference with MOLA MEGDR and PEDR is higher than all other strips. We can see from the graph that the heights are similar between each year, with a height difference of $\leq 1 \mathrm{~m}$ for $61.64 \%$ of the 
overlapping points $\left(2.18 \times 10^{7}\right.$ points from the total of $3.54 \times 10^{7}$ points $)$. Calculating the statistics without extreme values, we obtain a mean of differences of $2.3525 \mathrm{~m}$, and a root mean square difference of $48.3844 \mathrm{~m}$. The mean differences between overlapping DTMs are generally in agreement with the RMS errors in the data products (Table 2), which indicates that the vertical changes differences in the HRSC DTMs over this region between MY 27 and MY 28 is not an indication of annual changes, but an indication of bundle adjustment quality of individual HRSC image strips, as based on Smith, et. al. Smith et al. (2001b), the maximum yearly height differences over the south poles between two MYs are $1.5-2 \mathrm{~m}$.

Although seasonal changes are visible in the orthorectified images (such as shown in Figure 7 between H2183_0000 and H2372_0000 (top); as well as H4162_0000 and H4917_0009 (bottom)), this is unimportant for the DTM because the thickness of the seasonal ice cap is less than $1 \mathrm{~m}$ (Phillips, 2006)), which is smaller than the vertical precision of the HRSC DTMs. This can be shown by comparing different DTMs in overlapping areas with DTMs produced from different years (annual stability) and produced from the same year but with different solar longitudes (seasonal stability).

We can see small differences in the height comparison between DTMs made from images taken in different seasons for both MY27 and MY28 (see 8). We check for seasonal stability by differencing image strips which have a distinctive gap between seasons to increase the extrimity of surface differences. We use 13 strips for MY27 (8 strips from spring/ earlier sets of $\mathrm{L}_{\mathrm{s}} 5$ from the end of summer/ later sets of $\mathrm{L}_{\mathrm{s}}$ and for MY28, we used 4 image strips $(2$ strips from the start of spring / earlier sets of $\mathrm{L}_{\mathrm{s}}$ and 2 strips from the end of summer/ autumn, the later sets of $\mathrm{L}_{\mathrm{s}}$. We did not include the strips with in-between- $\mathrm{L}_{\mathrm{s}}$ value as we want to have the most surface changes from the HRSC image strips we used. We did not calculate seasonal stability for MY29 as there are only two image strips available in this research. The graph is presented in logarithmic scale because the percentage of height difference of $\leq 1 \mathrm{~m}$ is far higher compared to other height differences. From the graph, we could see that the majority of the discrete height points have a difference of $\leq 1 \mathrm{~m}\left(80.10 \%\right.$ for MY27, corresponding to $1.23 \times 10^{7}$ points from the total of $1.53 \times 10^{7}$ and $50.10 \%$ for MY $280.85 \times 10^{6}$ from the total of $1.68 \times 10^{6}$. Calculating the statistics, for MY27 we obtain a mean of difference of $-0.412 \mathrm{~m}$, a root mean square difference of $7.988 \mathrm{~m}$, and standard deviation of $7.978 \mathrm{~m}$. The mean differences between overlapping DTMs are generally agreeable with the RMS errors in the data products (Table 2). In the comparison of two DTMs from different seasons of MY28, a larger mean offset and root mean square error are observed compared to the values from MY27. However, this is likely due to changing surface properties (e.g. a dust storm (Piqueux et al., 2008)) that affect the feature-matching algorithm rather than an actual change in elevation. We obtain a $8.506 \mathrm{~m}$ mean difference (without extremes) with a root mean square error of $221.730 \mathrm{~m}$ and standard deviation of $221.561 \mathrm{~m}$. Further research on the seasonal differences effect on DTM production needs to be done with higher resolution DTMs such as CTX or HiRISE, but for these HRSC DTMs, the results are likely to be similar between different seasons. 

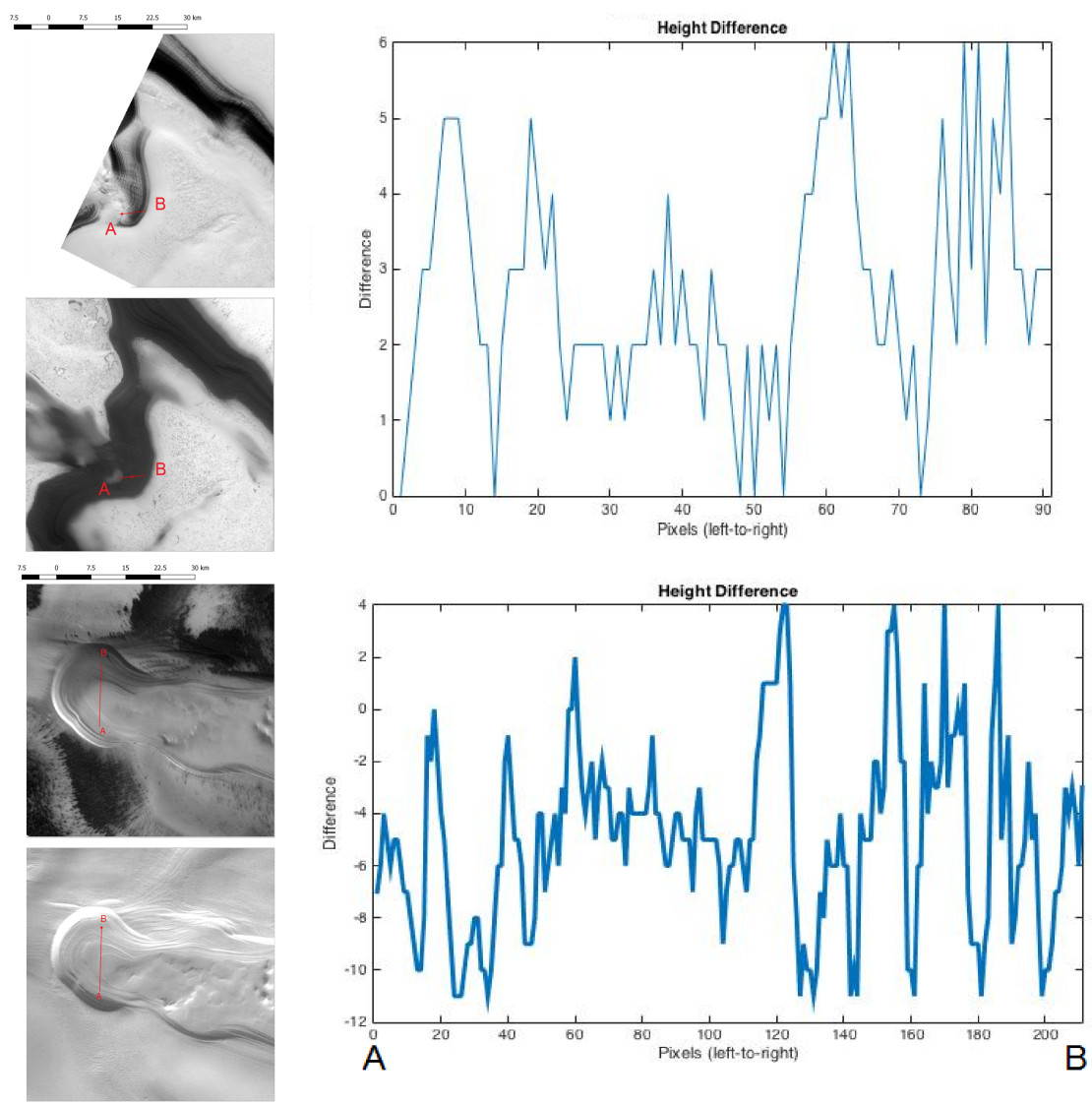

Figure 7: Seasonal stability testing by comparing height difference between seasons (top) (MY27) H2181_0000 (17-09-2005 L s $_{\text {s }}$ 294.6) bottom H2372_0000 (17-11-2005, L $\mathrm{s}_{\mathrm{s}}$ 325.7) (bot-

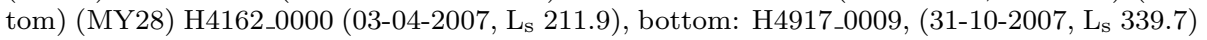




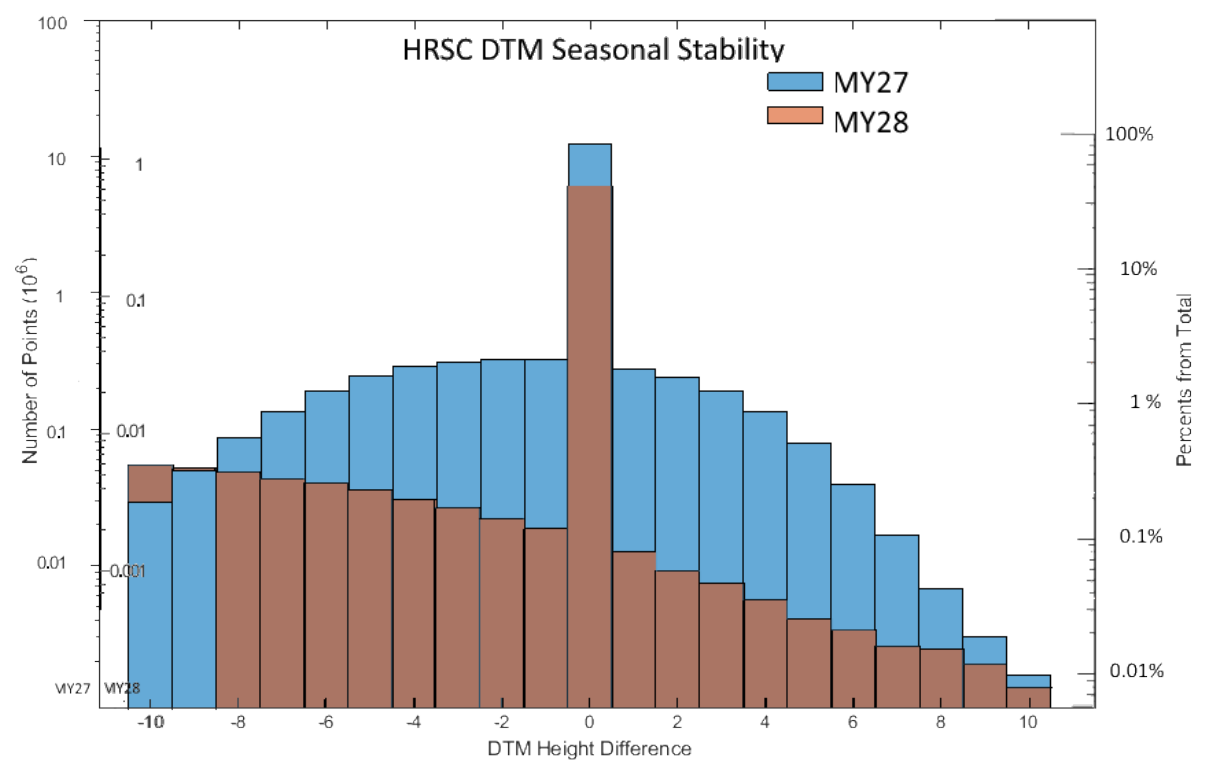

Figure 8: Seasonal stability testing by comparing height difference between seasons (biggest range of available $\mathrm{L}_{\mathrm{s}}$ ) for MY27(19 strips) and MY28(4 strips), not including H2287_0000, both tail not shown for clarity.

Further calculations on stability between DTMs can be obtained by making histograms of the height differences from grid points in overlapping DTM strips. From the histogram in Figure 9, we can see that the difference peaks at $-1 \mathrm{~m}$ then followed by $0 \mathrm{~m}$, which is in agreement with the results obtained by comparing single profiles, concluding that the DTM result is stable between different strips. Based on research on height changes obtained from MOLA measurement (Smith et al. 2001b), the interannual and intraannual changes on the south poles are shown to be less than $0.4 \mathrm{~m}$. Based on the annual and seasonal stability test calculated in this research, most errors in HRSC DTM (reflected in the height differences) arise from (a) lower resolution in the HRSC nadir images; (b) quality of the exterior orientations which depend greatly on dense feature points, whih are more difficult to obtain over snow and ice; (c) the lack of any bundle block adjustment such as the ones performed by Gwinner et al. Gwinner et al. (2016). Nevertheless, even given these limitations, the bias between overlapping strips for different MYs and Ls is very small (see 6 and 8), peaks at $-1 \mathrm{~m}$, as shown in 9

This planimetric correlation can also be seen by comparing the side-by-side appearances of orthorectified images around their edges. In the example, we can see that improved pointing from the exterior orientation worked well, shown by the smooth changes over the edges between different strips as pictured in Figure 10. Although the strips differ in appearance because of the differences in solar longitude value, the features are well aligned visually. If the difference in solar 


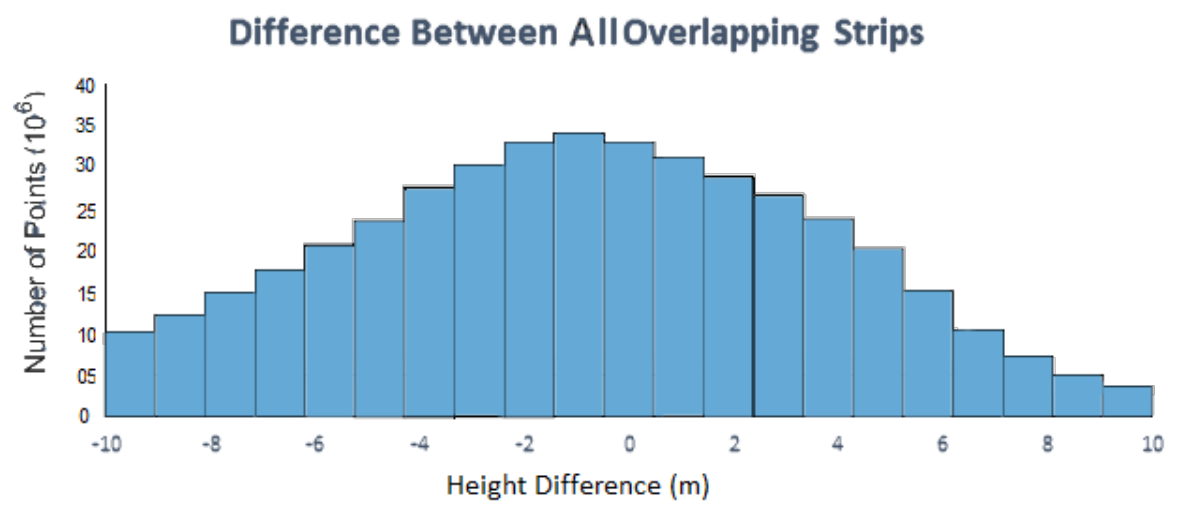

Figure 9: Differences between all overlapping strips in meters

longitude between strips is not much (adjacent strips are in the same season based on solar longitude), the strips can be seamlessly mosaicked to produce larger ORI products.

\subsection{Production of a Mosaic from Orthorectified Orbital strip Products}

Martian atmospheric variability makes it difficult to construct a seamless image mosaic. The presence of dust or aerosols in the camera's line of sight degrades the image quality, the fraction of light arriving directly from the surface diminishing with respect to that scattered by atmospheric dust particles. The consequence of a decrease in image contrast together with an increase in average brightness, leads to a 'patchwork' appearance when many images are compiled into a mosaic. It has proven difficult to make a systematic atmospheric correction, so we take an alternative approach: equalising the images to an external brightness reference (Michael et al., 2016). This enables retention of high resolution detail in the images while bringing the local absolute brightness of the images into mutual consistency. For the polar mosaic, we used a high altitude HRSC observation as the brightness reference: image HE150_0000 (2502-2015, MY33, $\mathrm{L}_{\mathrm{s}}$ 297.4), covering the whole of the cap with a swath width, in its vicinity, of around $2200 \mathrm{~km}$ and a surface resolution of about $440 \mathrm{~m} / \mathrm{pixel}$.

The changing ice cover at the polar cap introduces an additional complication to the mosaicking process. Ideally, we would choose a set of images taken over a short period, or from different years at similar Ls. Our selected images, chosen for their quality, however, cover a range of $120^{\circ}$ of Ls, equivalent to a third of a year. Consequently, we see some image boundaries where the ice coverage has shifted. Likewise, there are areas where the image is not entirely consistent with the brightness reference image (taken about 1/12 Martian year after southern midsummer), causing mild brightness artefacts at the edge of the cap.

The images from the 33 selected observations were initially sorted in order of ground sampling distance, compiling the lowest surface resolution image into the 


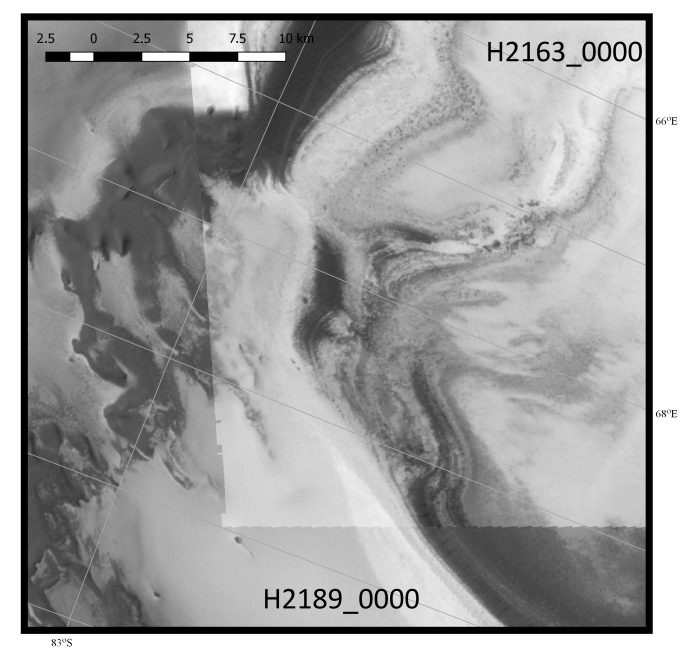

Figure 10: Examples of good co-registration between strip edges by comparing H2163_0000, 20-09-2005, MY27, L 291.64 with H2189_0000, 27-09-2005, MY27, L 296.05

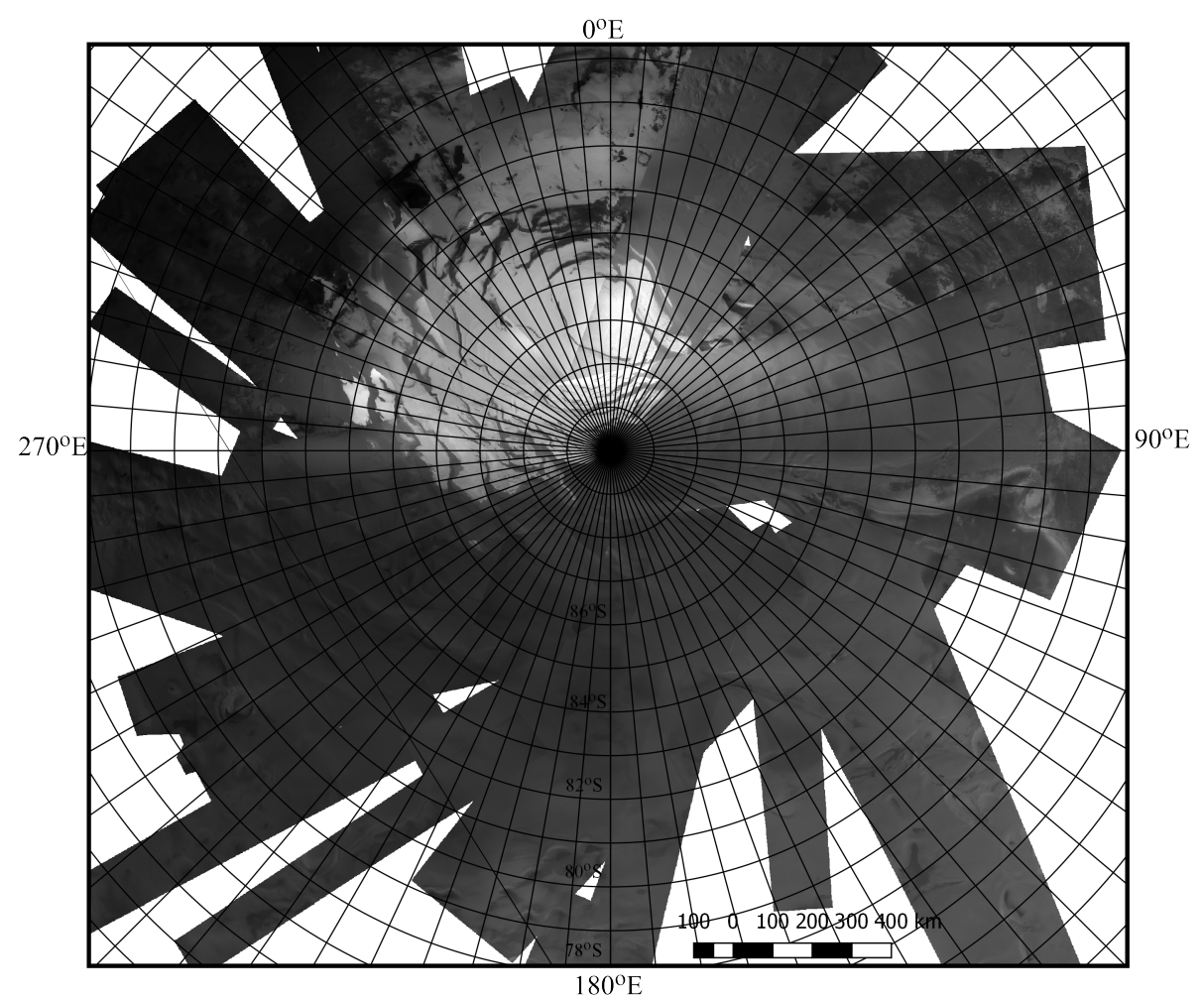

Figure 11: Mosaic of HRSC Orthorectified Images over the South Pole 


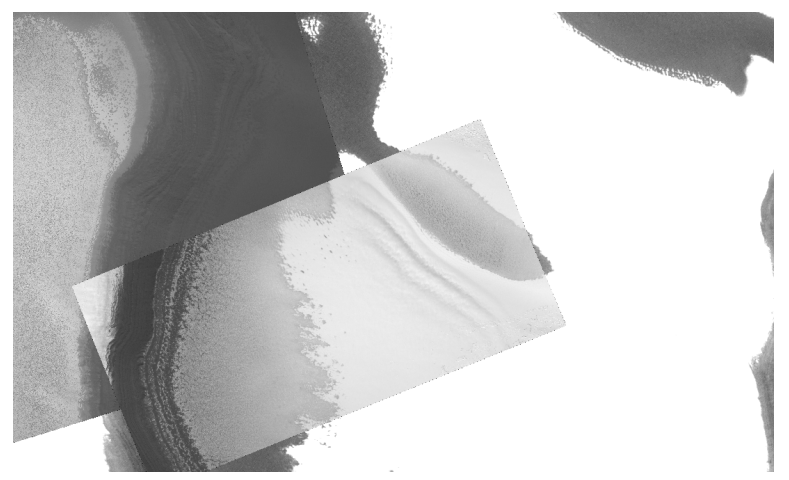

Figure 12: A mosaic of 2 CTX images and an HRSC ORI. The CTX images were co-registered to the HRSC ORI using the algorithm of (?). The image in the top-left part of the figure is CTX image D14_032511_0959_XI_84S078W_ORI (03-07-2013, MY31, Ls 345.80) while the image in the centre is the CTX image B11_013813_0955_XN_84S078W (07-07-2009, MY29, Ls 299.19). The background image is the level-4 HRSC ORI H2288_0000 (25-10-2005, MY27, $\mathrm{L}_{\mathrm{s}}$ 312.46). All images are demonstrated using polar stereographic projection

mosaic first, and the highest resolution last. This leaves the best resolved images at the top of the mosaic stack, and thus most visible in the final product. After inspection of the completed mosaic in $12.5 \mathrm{~m} /$ pixel, in order to not sacrifice the higher-resolution data, some alterations to the mosaicking sequence were made to move images with more strongly visible atmospheric artefacts lower in the sequence and optimise the quality of the final product (Figure 11).

\subsection{Automated co-registration and orthorectification (ACRO) of NASA High- Resolution South Pole Imagery}

The HRSC level-4 products that were generated using the pipeline presented in Section 4 are high-resolution 3D surface models with an inherent potential to facilitate the examination and analysis of South Polar dynamic surface phenomena. Their value is further increased by the use of the automated co-registration and orthorectification (ACRO) of NASA high-resolution South Pole imagery using the produced HRSC DTMs and ORIs as a baseline. The goal of this additional pipeline is to geometrically align all high-resolution South Pole imagery, but the final objective is to automatically detect South Pole surface changes through pixel-scale pairwise comparisons as well as multi-source data fusion. Preliminary results 12 indicate that there is no significant difference in the accuracy and reliability of the ACRO pipeline when the products derived from this work are used instead of those released by DLR (Gwinner et al., 2009), which provides further evidence for the quality and geometric accuracy of our new data products. More information about the ACRO algorithm can be found in Sidiropolous and Muller Sidiropoulos et al. (2018). 


\section{Conclusions and Future Work}

In this paper we have described the production of 33 HRSC orbital strip DTMs covering the SPRC and the South Polar region of Mars $\left(82^{\circ} \mathrm{S}-90^{\circ} \mathrm{S}\right)$ by using a method based on DLR-VICAR with modifications by Kim and Muller (2009). The DTMs produced show elevation differences of $-2.20 \mathrm{~m}$ (up to 0.04 $\mathrm{m}$ ) mean with MOLA PEDR points with a root mean square error of $-9.48 \mathrm{~m}$ and alternatively a mean difference of $1.08 \mathrm{~m}$ and $7.29 \mathrm{~m}$ root mean square error with MOLA MEGDR rasters. By comparing different strips taken in different seasons and different years, we find that there are no vertical changes larger than the errors in the DTMs. Different DTM strips can be merged into a single DTM mosaic based on an assessment of the height differences between overlapping strips. The produced ORIs are currently being used as base images for coregistering higher resolution images from CTX and the produced DTMs are currently being used as base DTM to produce higher resolution DTMs.

\section{Acknowledgements}

The first author acknowledges support for her studies from the Indonesian Endowment Fund of Education. This work forms part of the European Unions Seventh Framework Programme under iMars grant No. 607379 and by the German Space Agency (DLR Bonn), grant 50QM1702 (HRSC on Mars Express). Partial funding was obtained from the STFC MSSL Consolidated Grant ST/K000977/1. Green Open Access Funding is provided by University College London. The authors acknowledge the Principal Investigator, Ralf Jaumann, of the HRSC instrument onboard the Mars Express mission for providing datasets in the archive. Level-2 Input Datasets of the HRSC instrument have been downloaded from the ESA Planetary Science Archive (http://archives.esac.esa.int/psa). We also express gratitude to the HRSC team and the MOLA team for the usage of HRSC and MOLA data. In addition, the authors would like to thank Marita Wählisch for kind assistance with the areoid conversion and last but not least, Alexander Dumke for the exterior orientation processing results used within this work. The HRSC orbital strip and mosaiced products of the DTMs and ORIs which are described in this paper are available from the ESA Guest Storage Facility which can be found using the DOI of the HRSC data colection as follows: They can also be browsed, downloaded and visualised through the iMars web GIS at http://www.i-mars.eu/web-gis

\section{References}

Becker, K. J., Archinal, B. A., Hare, T. H., Kirk, R. L., Howington-Kraus, E., Robinson, M. S., Rosiek, M. R., 2015. Criteria for Automated Identification of Stereo Image Pairs. In: Lunar and Planetary Science Conference. Vol. 46 of Lunar and Planetary Inst. Technical Report. p. 2703. 
Blasius, K. R., Vetrone, A. V., Martin, M. D., 1980. Viking orbiter stereo imaging catalog. Tech. rep.

Bostelmann, J., Heipke, C., 2016. Global Bundle Adjustment with Variable Orientation Point Distance for Precise Mars Express Orbit Reconstruction. ISPRS - International Archives of the Photogrammetry, Remote Sensing and Spatial Information Sciences XLI-B4, 361-368.

Buhler, P. B., Ingersoll, A. P., Ehlmann, B. L., Fassett, C. I., Head, J. W., 2017. How the Martian Residual South Polar Cap Develops Quasi-Circular and Heart-Shaped Pits, Troughs, and Moats. Icarus 286, 69-93.

Byrne, S., Ingersoll, A. P., 2003. A Sublimation Model for Martian South Polar Ice Features. Science 299 (5609), 1051-1053. URL http://science.sciencemag.org/content/299/5609/1051

Calvin, W., Cantor, B., James, P., 2017. Interannual and Seasonal Changes in The South Seasonal Polar Cap of Mars: Observations from MY 28-31 using MARCI. Icarus 292, 144-153.

Cantor, B. A., Wolff, M. J., James, P. B., Higgs, E., 1998. Regression of Martian North Polar Cap: 19901997 Hubble Space Telescope Observations. Icarus 136 (2), 175-191.

Christensen, P. R., Jakosky, B. M., Kieffer, H. H., Malin, M. C., McSween Jr, H. Y., Nealson, K., Mehall, G. L., Silverman, S. H., Ferry, S., Caplinger, M., 2004. The Thermal Emission Imaging System (THEMIS) for the Mars 2001 Odyssey Mission. Space Science Reviews 110 (1-2), 85-130.

Duxbury, T. C., Kirk, R. L., Archinal, B. A., Neumann, G. A., 2002. Mars Geodesy/Cartography Working Group Recommendations on Mars Cartographic Constants and Coordinate Systems. International Archives of Photogrammetry Remote Sensing and Spatial Information Sciences 34 (4), 743748.

Geissler, P. E., 2005. Three Decades of Martian Surface Changes. Journal of Geophysical Research 110 (E2).

Gruen, A., 1985. Adaptive Least Squares Correlation: A Powerful Image Matching Technique. South African Journal of Photogrammetry, Remote Sensing and Cartography 14 (3), 175-187.

Gwinner, K., Jaumann, R., Hauber, E., Hoffmann, H., Heipke, C., Oberst, J., Neukum, G., Ansan, V., Bostelmann, J., Dumke, A., Elgner, S., Erkeling, G., Fueten, F., Hiesinger, H., Hoekzema, N., Kersten, E., Loizeau, D., Matz, K.D., McGuire, P., Mertens, V., Michael, G., Pasewaldt, A., Pinet, P., Preusker, F., Reiss, D., Roatsch, T., Schmidt, R., Scholten, F., Spiegel, M., Stesky, R., Tirsch, D., van Gasselt, S., Walter, S., Whlisch, M., Willner, K., 2016. The High Resolution Stereo Camera (HRSC) of Mars Express andIts Approach 
to Science Analysis and Mapping for Mars and Its Satellites. Planetary and Space Science 126, 93-138.

Gwinner, K., Scholten, F., Preusker, F., Elgner, S., Roatsch, T., Spiegel, M., Schmidt, R., Oberst, J., Jaumann, R., Heipke, C., 2010. Topography of Mars from Global Mapping by HRSC High-Resolution Digital Terrain Models and Orthoimages: Characteristics and Performance. Earth and Planetary Science Letters 294 (3), 506-519.

Gwinner, K., Scholten, F., Spiegel, M., Schmidt, R., Giese, B., Oberst, J., Heipke, C., Jaumann, R., Neukum, G., 2009. Derivation and Validation of High-Resolution Digital Terrain Models from Mars Express HRSC Data. Photogrammetric Engineering \& Remote Sensing 75 (9), 1127-1142.

Heipke, C., Oberst, J., Albertz, J., Attwenger, M., Dorninger, P., Dorrer, E., Ewe, M., Gehrke, S., Gwinner, K., Hirschmller, H., others, 2007. Evaluating Planetary Digital Terrain ModelsThe HRSC DTM Test. Planetary and Space Science 55 (14), 2173-2191.

Herschel, W., 1784. XIX. On The Remarkable Appearances at The Polar Regions of The Planet Mars, and Its Spheroidical Figure; with A Few Hints Relating to Its Real Diameter and Atmosphere. Philosophical Transactions of the Royal Society of London 74, 233-273.

James, P. B., 1979. Recession of Martian North Polar Cap: 19771978 Viking Observations. Journal of Geophysical Research: Solid Earth 84 (B14), 83328334 .

James, P. B., Briggs, G., Barnes, J., Spruck, A., 1979. Seasonal Recession of Mars' South Polar Cap as Seen by Viking. Journal of Geophysical Research: Solid Earth 84 (B6), 2889-2922.

John Louis Emil, D., 2013. The Scientific Papers of Sir William Herschel. Vol. 1. Cambridge University Press.

Kieffer, H. H., 2007. Cold Jets in The Martian Polar Caps. Journal of Geophysical Research: Planets 112 (E8).

Kim, J., Lin, S.-Y., Choi, Y.-S., Kim, Y.-H., 2013. Toward Generalized Planetary Stereo Analysis SchemePrototype Implementation with Multi-Resolution Martian Stereo Imagery. Earth, Planets and Space 65 (7), 799-809.

Kim, J., Muller, J.-P., 2009. Multi-Resolution Topographic Data Extraction from Martian Stereo Imagery. Planetary and Space Science 57 (14-15), 20952112.

Kirk, R., Howington-Kraus, E., Rosiek, M., Anderson, J., Archinal, B., Becker, K., Cook, D., Galuszka, D., Geissler, P., Hare, T., et al., 2008. Ultrahigh Resolution Topographic Mapping of Mars with MRO HiRISE Stereo Images: Meter-Scale Slopes of Candidate Phoenix Landing Sites. Journal of Geophysical Research: Planets 113 (E3). 
Kirk, R., Soderblom, L., Howington-Kraus, E., Archinal, B., Team, A., 2002. USGS High Resolution Topo-Mapping of Mars with Mars Orbiter Camera Narrow-Angle Images. International Archives of Photogrammetry Remote Sensing and Spatial Information Sciences 34 (4), 713-722.

Lemoine, F. G., Smith, D. E., Rowlands, D. D., Zuber, M. T., Neumann, G., Chinn, D., Pavlis, D., 2001. An Improved Solution of the Gravity Field of Mars(GMM-2 B) from Mars Global Surveyor. Journal of Geophysical Research 106 (E10), 23-359.

Malin, M. C., Edgett, K. S., Cantor, B. A., Caplinger, M. A., Danielson, G. E., Jensen, E. H., Ravine, M. A., Sandoval, J. L., Supulver, K. D., 2010. An Overview of the 1985-2006 Mars Orbiter Camera Science Investigation. International Journal of Mars Science and Exploration 5, 1-60.

Mattson, S., Kirk, R. L., Heyd, R., McEwen, A. S., Eliason, E., Hare, T., Beyer, R., Howington-Kraus, E., Okubo, C., Herkenhoff, K., 2011. Release of HiRISE Digital Terrain Models to the Planetary Data System. Vol. 42. p. 1558.

Michael, G. G., Walter, S. H. G., Kneissl, T., Zuschneid, W., Gross, C., McGuire, P. C., Dumke, A., Schreiner, B., van Gasselt, S., Gwinner, K., et al., 2016. Systematic Processing of Mars Express HRSC Panchromatic and Colour Image Mosaics: Image Equalisation using an External Brightness Reference. Planetary and Space Science 121, 18-26.

Neukum, G., Jaumann, R., 2004. HRSC: The High Resolution Stereo Camera of Mars Express. In: Mars Express: The Scientific Payload. Vol. 1240. pp. $17-35$.

Otto, G. P., Chau, T. K. W., 1989. Region-Growing Algorithm for Matching of Terrain Images. Image and vision computing 7 (2), 83-94.

Phillips, T., 2006. Mars is Melting. Science @ NASA.

URL https://science.nasa.gov/science-news/science-at-nasa/2003/ 07aug_southpole

Piqueux, S., Byrne, S., Richardson, M. I., 2003. Southern Seasonal CO Ice Cap and the Formation of Spiders. Journal of Geophysical Research: Planets 108 (E8).

Piqueux, S., Edwards, C. S., Christensen, P. R., 2008. Distribution of the ices exposed near the south pole of mars using thermal emission imaging system (themis) temperature measurements. Journal of Geophysical Research: Planets 113 (E8).

Piqueux, S., Kleinböhl, A., Hayne, P. O., Kass, D. M., Schofield, J. T., McCleese, D. J., 2015. Variability of The Martian Seasonal CO2 Cap Extent over Eight Mars Years. Icarus 251, 164-180. 
Poole, W., Muller, J.-P., Gupta, S., Grindrod, P. M., 2014. Calibrating Mars Orbiter Laser Altimeter Pulse Widths at Mars Science Laboratory Candidate Landing Sites. Planetary and Space Science 99, 118-127.

Scholten, F., Gwinner, K., Roatsch, T., Matz, K.-D., Whlisch, M., Giese, B., Oberst, J., Jaumann, R., Neukum, G., 2005. Mars Express HRSC Data Processing Methods and Operational Aspects. Photogrammetric Engineering \& Remote Sensing 71 (10), 1143-1152.

Seidelmann, P. K., Abalakin, V. K., Bursa, M., Davies, M. E., De Bergh, C., Lieske, J. H., Oberst, J., Simon, J. L., Standish, E. M., Stooke, P., 2002. Report of the IAU/IAG Working Group on Cartographic Coordinates and Rotational Elements of The Planets and Satellites: 2000. Celestial Mechanics and Dynamical Astronomy 82 (1), 83-111.

Sheehan, W., 1996. The Planet Mars: A History of Observation \& Discovery. University of Arizona Press.

Shin, D., Muller, J.-P., 2012. Progressively Weighted Affine Adaptive Correlation Matching for Quasi-Dense 3D Reconstruction. Pattern Recognition 45 (10), 3795-3809.

Sidiropoulos, P., Muller, J.-P., 2018. A Systematic Solution to Multi-Instrument Co-Registration to High-Resolution Planetary Images to an Orthorectified Baseline. IEEE Transactions on Geoscience and Remote Sensing 561 (1), 7892 .

Sidiropoulos, P., Muller, J.-P., Watson, G., Michael, G., Walter, S., 2018. Automatic Coregistration and Orthorectification (ACRO) and Subsequent Mosaicing of NASA High-Resolution Imagery over The Mars MC11 Quadrangle, using HRSC as A Baseline. Planetary and Space Science 151, 33 - 42.

Smith, D., Neumann, G., Ford, P., Arvidson, R. E., Guinness, E. A., Slavney, S., 1999. Mars Global Surveyor Laser Altimeter Mission Experiment Gridded Data Record, MGS-M-MOLA-3-PEDR-L1A-V1.0. NASA Planetary Data System.

Smith, D. E., Zuber, M. T., Frey, H. V., Garvin, J. B., Head, J. W., Muhleman, D. O., Pettengill, G. H., Phillips, R. J., Solomon, S. C., Zwally, H. J., et al., 2001a. Mars orbiter laser altimeter: Experiment summary after the first year of global mapping of mars. Journal of Geophysical Research: Planets 106 (E10), 23689-23722.

Smith, D. E., Zuber, M. T., Neumann, G. A., 2001b. Seasonal Variations of Snow Depth on Mars. Science 294 (5549), 2141-2146.

URL http://science. sciencemag . org/content/294/5549/2141

Soffen, G. A., Snyder, C. W., 1976. The First Viking Mission to Mars. Science 193, 759-766. 
Tao, Y., Muller, J.-P., 2017. Automated Planet-Wide DTM Generation from NASA MRO Data A Status Report. In: Lunar and Planetary Science Conference. Vol. 48 of Lunar and Planetary Institute Technical Report. p. 2965.

Tao, Y., Muller, J.-P., Sidiropoulos, P., Xiong, S.-T., Putri, A., Walter, S., Veitch-Michaelis, J., Yershov, V., 2018. Massive Stereo-Based DTM Production for Mars on Cloud Computers. Planetary and Space Science.

Thomas, N., Cremonese, G., Banaszkiewicz, M., Bridges, J., Byrne, S., da Deppo, V., Debei, S., El-Maarry, M. R., Hauber, E., Hansen, C. J., 2014. The Colour and Stereo Surface Imaging System (CaSSIS) for ESA's Trace Gas Orbiter. In: 8th International Conference on Mars.

Thomas, P., Calvin, W., Cantor, B., Haberle, R., James, P., Lee, S., 2016. Mass Balance of Mars Residual South Polar Cap from CTX Images and Other Data. Icarus 268, 118-130.

Thomas, P., James, P., Calvin, W., Haberle, R., Malin, M., 2009. Residual South Polar Cap of Mars: Stratigraphy, History, and Implications of Recent Changes. Icarus 203 (2), 352-375.

Thomas, P., Malin, M., James, P., Cantor, B., Williams, R., Gierasch, P., 2005. South Polar Residual Cap of Mars: Features, Stratigraphy, and Changes. Icarus 174 (2), 535-559.

Thomas, P. C., Malin, M. C., Edgett, K. S., Carr, M. H., Hartmann, W. K., Ingersoll, A. P., James, P. B., Soderblom, L. A., Veverka, J., Sullivan, R., 2000. NorthSouth Geological Differences Between the Residual Polar Caps on Mars. Nature 404 (6774), 161-164.

Thornhill, G. D., Rothery, D. A., Murray, J. B., Cook, A. C., Day, T., Muller, J.-P., Iliffe, J., 1993. Topography of Apollinaris Patera and Ma'adim Vallis: Automated Extraction of Digital Elevation Models. Journal of Geophysical Research: Planets 98 (E12), 23581-23587. 